Case Report

\title{
A Submandibular Sialolith of an Unusual Size Presenting With a Cervical Fistula M imicking a Branchial Fistula : A Case Report
}

Rajesh Ballal ${ }^{1}$, Khamarjaha Banu' ${ }^{2} \&$ Kalpa Pandya ${ }^{3}$

${ }^{1,2}$ Professors, Department of General Surgery, K. S. Hegde Medical Academy,

${ }^{3}$ Post Graduate, Department of Oral \& M axillofacial Surgery, A.B. Shetty Memorial Institute of Dental Sciences, Nitte University, Mangalore, Karnataka, India.

*Corresponding Author : Kalpa Pandya, Post Graduate, Department of Oral \& Maxillofacial Surgery, A.B. Shetty Memorial Institute of Dental Sciences, Nitte University, M angalore - 575 018, Karnataka, India.

Mobile: +919632165530 E-mail : kalpa.pandya@gmail.com

Received

:20-04-2015

Review Completed : 18-01-2016

Accepted

: 01-03-2016

Keywords : submandibular gland; calculus; sialadenitis; fistula

\begin{tabular}{|c|}
\hline Access this article online \\
\hline Quick Response Code \\
\hline
\end{tabular}

\begin{abstract}
:
Sialo liths greater than $10 \mathrm{~mm}$ in size can be called as those of an unusual size. Only $7.65 \%$ of the sialoliths are $>15 \mathrm{~mm}$. Cutaneous fistulas due to submandibular sialoliths are even rarer. A case of a submandibular gland sialolith of an unusual size presenting with a cervical fistula mimicking a branchial fistula has been reported. Intra-operatively, the fistulous tract was found to extend upto the supraclavicular space. Complete excision of the gland along with fistulous tract was performed. Histopathology revealed a sialolith with acute and chronic sialedinitis. The lesion also mimicked a Kuttnor's tumour however a final diagnosis of the same could not be made due to inconclusive Ig $G$ value and absence of plasmacytic infiltration.
\end{abstract}

\section{Introduction}

Sialolithiasis is the formation of a calcified mass (sialolith) in the salivary glands. It is said to affect 0.1 to $1 \%$ of the population with a male predilection and 30 to 60 years being the most common age. (1)Of all the salivary glands, sialoliths are most commonly found in the submandibular gland $(80-95 \%)$ (2). This is owing to longer, tortuous Wharton's duct, anti-gravity flow of saliva in submandibular gland and a more viscous consistency of the submandibular secretion due to alkalinity, mucin proteins and greater amount of calcium and phosphates. (3)According to Lutsmann et al, $78.8 \%$ of sialoliths are $<10$ $\mathrm{mm}, 13.6 \%$ are between $10-15 \mathrm{~mm}$ and $7.65 \%$ are $>15 \mathrm{~mm}$. They more commonly found in the duct than in the parenchyma. (4)

Sialadenitis is the inflammation of the salivary glands. The etiology can be infectious or autoimmune. Incidence of sialadenitis in submandibular gland consists of $10 \%$ of all major salivary glands. Silaolithiasis is considered to be one of the common causes of sialadenitis. Sialadenitis commonly shows an enlarged, tender gland causing pain on swallowing and elevation of the floor of the mouth.

A cutaneous fistula seen in the neck along the anterior border of the sternocleidomastoid is a common presentation of second or third branchial cleft fistula or sinus. Although this condition is congenital, it may present later in life. (5) The presentation is however rare after 40 years of age. A hetertotropic salivary gland may also present similarly however being rare. (6) A fistula in the neck as a result of sialadenitis and a sialolith of an unusual size, extending upto the supraclavicular space and showing a fistulous opening in the middle of neck could not be 
found in the English literature.

We report a case of a sialolith of an unusual size in the submandibular gland presenting with sialadenitis manifesting as a fistula in the cervical region mimicking a branchial fistula.

\section{Case Report}

An 80 year old male reported to Justice K.S. Hegde M edical Hospital with the chief complaint of pain and pus discharge from the left side of the neck since 15 years. The pain was insidious in onset, gradually progressive, and of throbbing type. The patient was a known case diabetes mellitus type II, hypertension and asthma. Patient gave a history of incision and drainage being done in the same region 5 years back.

On examination an extraoral swelling was noted in the left submandibular region which was hard and localised on bimanual palpation. No tenderness or discomfort was noted. A cutaneous fistulous opening was visible in the neck roughly at the junction of upper third and lower two third of the anterior border of the sternocleidomastoid. This presentation was similar to that due to a branchial fistula. (Fig 1-A \& B) Intraoral examination revealed an edentulous mandible however no swelling or signs were noted in relation to the floor of the mouth or the opening of the submandibular duct. A CT scan was done which revealed a large sialolith of the left submandibular gland and a fistulous tract extending from the submandibular gland. (Fig 2) A provisional diagnosis of chronic sialadenitis manifesting as a cervical fistula in association with a large sialolith was made.

The patient was taken up for sialedenectomy and neck exploration under general anesthesia. A horizontal incision was first made involving the fistula. Surprisingly, when the fistulous tract was traced, it led to the left supraclavicular area. A small incision was made in the supraclavicular area which confirmed the extension of the tract to the same area.(Figure 3) The tract here was disconnected.The tract was then traced above the fistulous opening which led to the submandibular gland. The extensive fibrosis of the periglandular tissue made the dissection difficult. The gland was then excised with careful dissection along with the fistulous tract. (Figure 4-A)Hemostasis was achieved and the wound was closed in layers. The gland revealed a large sialolith measuring 30X10X10 mm. (Figure 4- B) Histopathological examination revealed dilated ducts showing calculi with dense infiltration of leukocytes. It also showed dense infiltration of lymphocytes along with fibrosis. An informed consent was taken before publishing the case.

\section{Discussion}

Sialolithiasis accounts for approximately $30 \%$ of all salivary gland disorders. It normally presents with pain, swelling and tenderness. Sialedinitis more commonly involves parotid gland however submandibular gland may also be involved. It shows elevation of the floor of the mouth, pain, tenderness and swelling. In the present case, the patient was relatively asymptomatic in relation to the submandibular region. The fistulous opening present in the cervical region was associated with pain and minimal discharge. The common region for the occurrence of sialolith includes the duct especially near the curved portion and the hilum. Intraparenchymal sialoliths are rare. (7) In the present case the stone was found within the parenchyma of the gland which accounts for only 9 to $17 \%$ as reported by the literature. (4). The average size of the salivary calculi is known to lie between 3.3 and $17.9 \mathrm{~mm}$ (8)and all sialoliths greater than $10 \mathrm{~mm}$ in size can be called asthose of an unusual size (9).

A large sialolith or chronic sialidinitis have been known to create opening in the floor of the mouth considering the fact that infection is known to follow the path of least resistance (10). However in the current case, the infection has spread along the superficial layer of the deep cervical fascia or rather below it,reaching the supraclavicular space. The superficial layer of the deep cervical fascia envelopes the parotid and the submandibular gland; sternocleidomastoid and trapezius muscle creating the suprasternal space of Burns above the sternum. Cutaneous fistulas due to submandibular sialoliths are rare. (11) 
(12) (13)No evidence of cervical fistula leading to the supraclavicular space due to an unusual size submandibular sialolith presenting in the parenchyma of the glands has ever been reported in the English literature.

The conventional way to treat a sialolith includes removal of the sialolith through an intraoral incision. Owing to the unusual size of the sialolith in the parenchyma of the gland and presence of the fistulous tract a decision to perform sialoadenectomy was made.

A literature review of sialoliths demonstrate a rather underdiagnosed condition known as Kuttner's tumour or chronic sclerosing sialadenitis. It has been classified under tumor like lesions of salivary glands by the World Health Organisation (WHO). (14)The condition is known to show a hard asymptomatic swelling in the parenchyma of the gland, the gland showing fibrosis, dense lymphoplasmacytic infiltration and loss of acini. The

\section{References}

1. Andretta M, Tregnaghi A, Prosenikliev V and Staffieri A:Current opinions in sialolithiasis diagnosis and treatment. Acta Otorhinolaryngol Ital:2005 and 145-149., 25:.

2. Endod.2002, Bodner L: Giant salivary gland calculi: Diagnostic imaging andsurgical management. Oral Surg Oral Med Oral Pathol OralRadiol and $94: 320$.

3. Raksin SZ, Gould SM, William AC. Submandibular gland sialolithof unusual size and shape. J oral Surg 1975 and 142-45., 33:

4. Lustmann J, Regev E and Melamed Y: Sialolithiasis. A survey on245 patients and a review of the literature. Int J Oral M axillofac Surg.1990 and 19:135-138.

5. Sampath P., Azeez A.,Dinaker T.Branchial Anomalies: Diagnosis and Management. Int J Otolaryngol. 2014 and 4, 2014:237015. doi: 10.1155/2014/237015. Epub 2014 M ar.

6. Jain S, Aggrawal A, Deshmukh P, Singhvi P, Sudarshan K. Heterotopic salivary glandpresenting as a discharging sinus in the base of the neck.Clinics and Practice 2011 and 1:e131.

7. Kopec T, Wierzbicka M, Szyfter W, Leszczynska M .Algorithm changes in treatment of submandibular gland.Eur Arch Otorhinolaryngol (2013) 270:2089-2093.

8. Anneroth G, Eneroth CM and Isacsson G: Morphology ofsalivary calculi. The distribution of the inorganic component. J Oral Pathol.1975 and 257-265., 4:. patients are also known to have a high serum IgG4. (15) On the basis of the clinical picture of the patient, a differential diagnosis of Kuttner's tumour was made and serum sent for IgG4evalution. The value of IgG4 was inconclusive and hence a definitive diagnosis as Kuttner's tumour could not be made. Also absence of any plasmcytic infiltration and presence of leukocytesin addition to lymphocytes histopathologically, further led to ruling out of this lesion.

\section{Conclusion}

Apart from conventional surgical treatment, treatment modalities such as sialedenoscopy and lithotripsy are gaining momentum. However for salivary stones greater than $12 \mathrm{~mm}$ and those which fail lithotripsy treatment, gland removal still remains a mainstay. (16) This case represents a unique finding of a cutaneous fistula mimicking a branchial fistula in addition to the presence of a sialolith of an unusual size in the parenchyma of the gland.

9. Batori M, Mariotta G, Chatelou H, et al: Diagnostic and surgical management of submandibular gland sialolithiasis: Report of a stone of unusual size. Eur Rev M ed Pharmacol Sc.2005 and 9:67., i.

10. Singh S, Singh S. Submandibular gland megalith eroding the floor of the mouth: a case report. Ear Nose Throat 20113 Dec and 92(12):E179.

11. Saha S, Jha A, Kaur N. Unusual Cause of Orocutaneous Fistula in the Neck. Case Rep Surg. 2012 and 658536., 2012:

12. Asfar SK, Steitiyeh MR, Abdul-Amir R. Giant salivary calculi: an orocervical fistula caused by a submandibular gland calculus. Can J Surg.1989 and 32(4):295-296.

13. Druez W, De Wolf JP, Tant L. Cutaneous fistula with lithiasis of the submaxillary gland. Acta Stomatol Belg. 1982 and 79(4):269-271.

14. 1992, Seifert G. Tumour-like lesions of the salivary glands.The new WHO classification. Path Res Pract and 188:836-846.

15. Chow T L, Chan T T, Choi C Y, Lam S H.Kuttner's tumour (chronic sclerosing sialadenitis) of the submandibular gland: a clinical perspective. Hong Kong M ed J.2008 Feb and 18:1.

16. Zenk J, Benzel W, Iro H. New modalities in the management of human sialolithiasis. M inimally nvasive therapy 1994 and 275-284., 3:.

17. Druez W, De Wolf JP, Tant L. Cutaneous fistula with lithiasis of the submaxillary gland. Acta Stomatologica Belgica. 1982 and 79(4):269-271. 